\title{
Causes and classification of male infertility in Korea
}

\author{
Hui Dai Lee, Hyo Serk Lee, Se Hwan Park, Dae Gi Jo, Jin Ho Choe, Joong Shik Lee, Ju Tae Seo \\ Department of Urology, Cheil General Hospital, Kwandong University College of Medicine, Seoul, Korea
}

Objective: The aim of this study is to investigate the various causes of male infertility using multiple approaches.

Methods: Nine-hundred-twenty infertile male patients were analyzed at their first visit with one physician between January 1 and December 31 , 2009. All patients were subjected to physical examination, semen analysis and azoospermic patients underwent hormonal testing, chromosomal tests, and testicular biopsy. Semen analysis was based on the definition of the World Health Organization.

Results: Among the 920 patients, 555 patients (60.3\%) had semen results within the normal range, 269 patients (29.2\%) within the abnormal range, and 96 (10.5\%) were diagnosed with azoospermia. Varicoceles were diagnosed in 84 of the 555 normal-range patients (15.1\%) and in 113 of the 269 abnormal-range patients (42.0\%). Of the 96 patients with azoospermia, 24 patients (25\%) were diagnosed with obstructive azoospermia, 68 patients (71\%) with non-obstructive azoospermia, and 4 patients (4\%) with retrograde ejaculation.

Conclusion: Various causes of male infertility have been reported and diverse treatment methods can be adopted for each cause. In this regard, research must be conducted on a larger number of patients to accurately assess the various causes of infertility in Korean patients and to investigate various infertility treatment methods.

Keywords: Male infertility; Varicocele; Azoospermia

\section{Introduction}

The World Health Organization (WHO) defines infertility as the inability to conceive a child after 1 year of regular, unprotected intercourse. Infertility currently affects approximately $15 \%$ of all couples, with an increase anticipated over the next 20 years. Approximately $50 \%$ of cases of infertility can be attributed to male factors [1]. The defects causing male infertility can be classified as related to the hypothalamus or pituitary gland, the testicles, or defective sperm delivery due to disorders in the penis or related sexual glands [2].

Azoospermia and abnormal semen parameters, such as a decrease in sperm count, are often observed in infertile men. Azoospermia can

Received: Aug 31, 2012 · Revised: Oct 25, 2012 · Accepted: Nov 29, 2012 Corresponding author: Ju Tae Seo

Department of Urology, Cheil General Hospital, Kwandong University College of Medicine, 17 Seoae-ro 1-gil, Jung-gu, Seoul 100-380, Korea

Tel: +82-2-2000-7585 Fax:+82-2-2000-7787 E-mail:jtandro@cgh.co.kr

This is an Open Access article distributed under the terms of the Creative Commons Attribution Non-Commercial License (http://creativecommons.org/licenses/by-nc/3.0/) which permits unrestricted non-commercial use, distribution, and reproduction in any medium, provided the original work is properly cited. be classified as obstructive azoospermia (OA) or non-obstructive azoospermia (NOA). The causes of OA can be further divided into congenital and acquired closure of the ejaculatory tract, which can occur anywhere along the spermatic cord and epididymis. The most common cause of $\mathrm{OA}$ is vasectomy [3]. However, it may also be caused by the epididymis, vas deferens, or ejaculatory duct pathology relating to genitourinary infection, iatrogenic injury during scrotal or inguinal surgical procedures, and congenital anomalies such as congenital bilateral agenesis of the vas deferens (CBAVD) [4]. NOA is caused by disorders related to sperm formation in the testicles and can be classified into hypospermatogenesis (HP), maturation arrest (MA), and Sertoli cell-only syndrome (SCO) according to the cell composition of the seminiferous tubules.

Although there are many causes of male infertility, researchers in Korea have not clearly analyzed the prevalence of each cause of male infertility. The aim of this study is to investigates the various causes of male infertility and attempts to take multiple approaches in examining the causes of infertility in males. 


\section{Methods}

This study included a total of 920 male patients with infertility who visited Cheil General Hospital between January 1st and December 31th 2009 and were cared for by a single physician. This study was a retrospective study using data from medical records. After an evaluation of the subjects' medical history, the subjects' physical condition and semen were also examined. A Prader orchidometer was used during the physical exam to measure testicular volume and Dubin and Amelar's method was used to verify the existence of a varicocele [5].

Semen analysis was performed after the patients had abstained from sexual intercourse for 3 to 5 days. Based on the WHO definition (4th ed. 1999), the semen was classified into 3 categories: normal range, abnormal range, or azoospermia. Patients diagnosed with azoospermia then underwent serum hormone tests, including testosterone, FSH, LH, chromosomal analysis with azoospermic factor microdeletion and a testicular biopsy. NOA was defined as the condition in which the testicular tissue test identified a sperm formation defect, without evidence of closure of the ejaculatory duct. The testicular tissue was classified into HP, MA, and SCO according to the cell composition of the seminiferous tubules. Subjects who had features of both MA and SCO were diagnosed with MA. OA was defined by normal spermatogenesis by testicular biopsy. A chromosomal analysis was performed to search for genetic abnormalities that might account for the azoospermia.

\section{Results}

The average age of patients was $35.3 \pm 8.7$. Among the 920 patients who visited the hospital for male infertility between January 1 and December 31, 2009, semen analysis revealed 555 patients (60.3\%) within the normal range, 269 patients (29.2\%), within the abnormal range, and 96 (10.5\%) with azoospermia.

Varicoceles were diagnosed in 84 out of 555 normal-range patients (15.1\%) and in 113 out of the 269 abnormal-range patients (42.0\%) (Table 1). Among the 96 patients who were diagnosed with azoospermia, 24 patients (25\%) were diagnosed with OA, 68 patients $(71 \%)$ were diagnosed with NOA, and the remaining 4 azoospermia patients (4\%) were found to have retrograde ejaculation. Among the 24 OA patients, 12 patients had obstruction of the vas deferens or epididymis, 10 showed CBAVD, and 2 patients were diagnosed with ejaculatory duct obstruction. Among the 68 NOA patients, 33 were diagnosed with $\mathrm{SCO}, 10$ with MA, 8 with $\mathrm{HP}$, and 17 showed chromosomal abnormalities. Forty-seven patients had chromosomal abnormalities: 47,XXY in 14 patients: 46,XX in 1 patient: and $Y$ chromosome microdeletions in 2 patients (Figure 1).

Table 1. Varicoceles in infertile men

\begin{tabular}{ll}
\hline Semen analysis & Varicocele $(\%)$ \\
\hline Normal range & $184 / 555(15.1)$ \\
Abnormal range & $113 / 269(42.0)$ \\
Total & $197 / 824(23.9)$
\end{tabular}

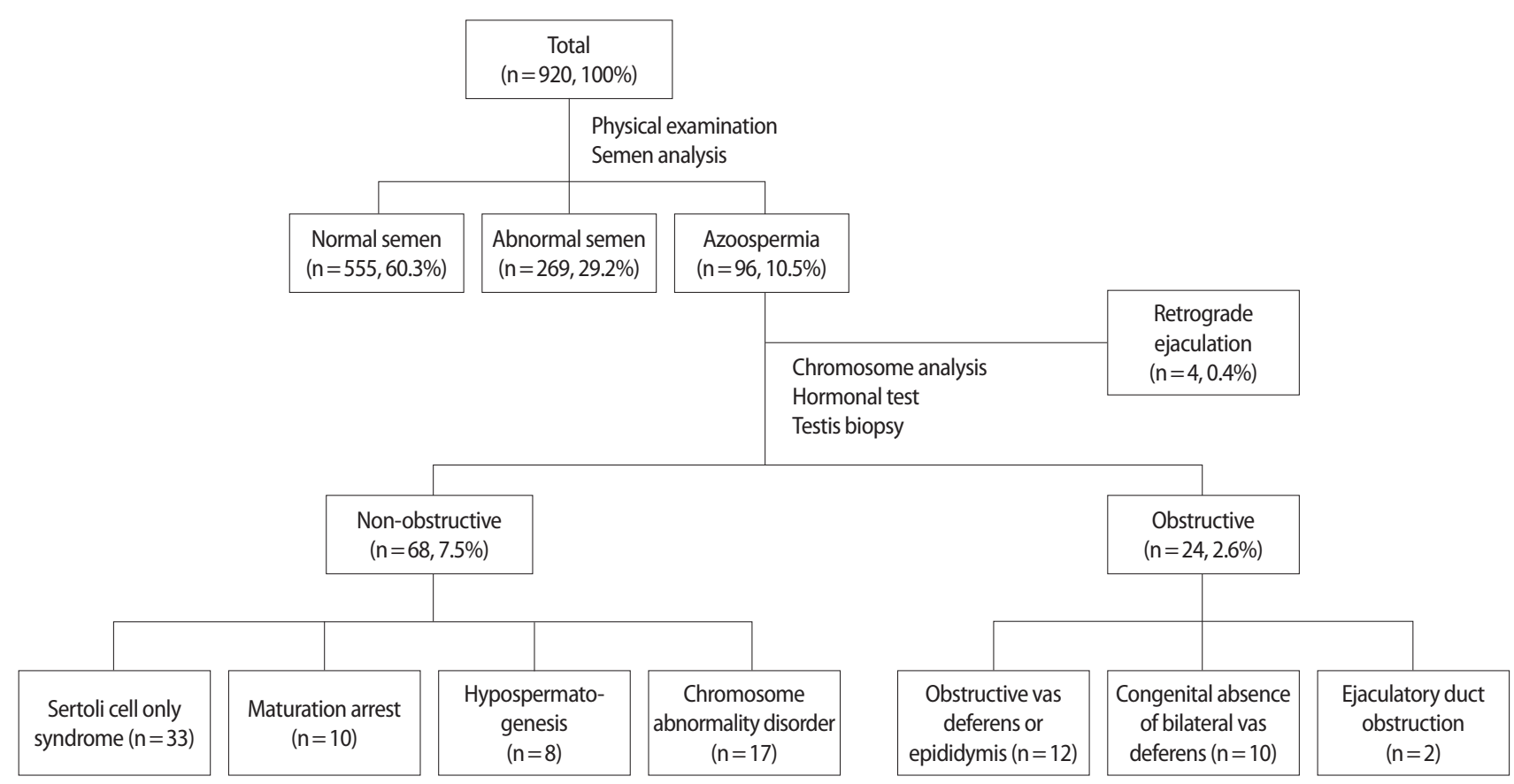

Figure 1. Patient distribution according to semen analysis results. 


\section{Discussion}

Male infertility is caused by various factors, such as disorders of the endocrine system, disturbances in ejaculation, bacterial or viral infection, immunologic factors, varicoceles, undescended testis and anabolic steroid use. However, a clear etiology cannot be determined for many cases of male infertility. Excluding patients who have previously had a vasectomy, nearly $10 \%$ to $15 \%$ of men have azoospermia, a condition in which semen is produced but does not contain sperm [6]. In this study, $60.3 \%$ had a normal range of semen parameters, $29.2 \%$ had an abnormal range of semen parameters and $10.5 \%$ had azoospermia. Azoospermia can be classified into $\mathrm{OA}$, a condition in which the sperm passage is congenitally or gradually closed, and NOA, in which there is disordered formation of sperm within the testicles. Another type of azoospermia is caused by disordered ejaculation. Forty percent of azoospermic cases are caused by post-testicular obstruction. Surgical treatment such as vasovasostomy or vasoepididymostomy should be considered for OA [7-10].

ICSI is a procedure that uses micromanipulation, which is commonly used during IVF. ICSI requires only one living sperm to achieve fertilization of one egg. It was demonstrated that this approach is superior to subzonal insemination after the first four human pregnancies by ICSI were reported by Palermo et al. [11]. This procedure presented a relatively high pregnancy success rate and opened a new era for treating infertility [11]. In the past, due to a lack of accreditation, ICSI was only implemented if regular external fertilization could not be achieved. This was often because the number of motile sperm in each ejaculated sperm was below 500,000 and the ratio of normal sperm was below $4 \%$. However, ICSI is now also being used in the following cases: 1 ) sperm count $<2 \times 10^{6} \mathrm{sperm} / \mathrm{mL}$; 2) sperm motility $<5 \%$; 3 ) normal morphology $<4 \%$ by strict criteria; 4 ) use of microsurgical epididymal sperm aspiration (MESA) or testicular sperm extraction (TESE) to acquire sperm, and 5) NOA such as MA and SCO [12-14].

Since treatment for azoospermia differs according to etiology, male patients with azoospermia should receive treatment based on accurate diagnosis and classification. This should lead to a high pregnancy success rate. According to previously reported data, 31\% of azoospermia is caused by obstructive factors, $54 \%$ is caused by non-obstructive factors generated by disorders in testicular function, and the remaining $15 \%$ appear normal without evidence of obstruction [15]. Among the 96 patients that were diagnosed with azoospermia in this study, 24 were OA patients (25\%), 68 were NOA (71\%), and the remaining 4 azoospermia patients (4\%) demonstrated retrograde ejaculation.

In treating CBAVD or OA, which cannot be treated surgically, successful fertilization and pregnancy can be achieved through MESA and ICSI [16]. Furthermore, if it is impossible to extract the sperm through
MESA due to obstruction, hypoplasia or surgical removal of epididymis, TESE and ICSI can be used instead to achieve sperm extraction [17]. Of those patients with NOA, $60 \%$ achieve pregnancy using ICSI after extracting a small amount of sperm through testicular biopsy. There are no treatment options for the remaining $40 \%$ even if multiple testicular sperm extraction operation were performed.

Varicocele is one of the most common causes of male factor infertility in patients that visit the hospital for infertility. Occurring in nearly $15 \%$ of male adults, varicocele is also found in $30 \%$ to $35 \%$ of primary infertility and $69 \%$ to $81 \%$ of secondary infertility $[18,19]$. Similar results about varicocele patients were observed in our study. Abnormal semen parameters in infertility patients with varicocele were first reported in 1965. At the same time, a connection between varicoceles and reduced sperm count and motility, testicular size and histological changes were also proposed [20].

In 1992, the WHO confirmed the correlation between varicoceles and reduced testicular volume, poor sperm quality and reduced Leydig cell function. Furthermore, several studies conducted on animal models and humans reported that varicoceles gradually trigger testicular damage depending on the length of disease [19,21,22].

Studies that have previously been carried out abroad have reported that the percent of varicoceles among patients with male infertility is $42.2 \%$ [23]. Varicoceles were diagnosed in 84 out of 555 infertility patients $(15.1 \%)$ who presented with semen analysis in the normal range and 113 out of 269 infertility patients (42.0\%) whose semen was in the abnormal range. When compared with the results of previous studies, the results of this study presented a similar percent of varicoceles causing abnormal semen analysis results.

Various causes of male infertility have been reported and diverse treatment methods can be adopted for each cause. In this study, the most common cause of male infertility was idiopathic. However, other causes of male infertility are also important to consider. In this regard, research must be conducted on a larger number of patients to accurately assess the various causes of infertility in Korean patients and to investigate various infertility treatment methods.

\section{Conflict of interest}

No potential conflict of interest relevant to this article was reported.

\section{REFERENCES}

1. Lee R, Li PS, Schlegel PN, Goldstein M. Reassessing reconstruction in the management of obstructive azoospermia: reconstruction or sperm acquisition? Urol Clin North Am 2008;35:289-301.

2. Korean Urological Associatioin. Urology. 3rd ed. Seoul: Korea Medical Book; 2001. 
3. Goldstein $M$, Tanrikut C. Microsurgical management of male infertility. Nat Clin Pract Urol 2006;3:381-91.

4. Practice Committee of American Society for Reproductive Medicine in collaboration with Society for Male Reproduction and Urology. The management of infertility due to obstructive azoospermia. Fertil Steril 2008;90:S121-4.

5. Dubin L, Amelar RD. Varicocele size and results of varicocelectomy in selected subfertile men with varicocele. Fertil Steril 1970; 21:606-9.

6. Jarow JP, Espeland MA, Lipshultz LI. Evaluation of the azoospermic patient. J Urol 1989;142:62-5.

7. Belker AM, Thomas AJ Jr, Fuchs EF, Konnak JW, Sharlip ID. Results of 1,469 microsurgical vasectomy reversals by the Vasovasostomy Study Group. J Urol 1991;145:505-11.

8. Schlegel PN, Goldstein M. Microsurgical vasoepididymostomy: refinements and results. J Urol 1993;150:1165-8.

9. Tanrikut C, Goldstein M. Obstructive azoospermia: a microsurgical success story. Semin Reprod Med 2009;27:159-64.

10. Kolettis PN, Thomas AJ Jr. Vasoepididymostomy for vasectomy reversal: a critical assessment in the era of intracytoplasmic sperm injection. J Urol 1997;158:467-70.

11. Palermo GD, Cohen J, Alikani M, Adler A, Rosenwaks Z. Intracytoplasmic sperm injection: a novel treatment for all forms of male factor infertility. Fertil Steril 1995;63:1231-40.

12. Seo JT, Park YS, Kim JH, Lee YS, Jun JH, Lee HJ, et al. The treatment of non-obstructive azoospermia. Korean J Fertil Steril 1997;24: 95-9.

13. Schlegel PN. Techniques of assisted reproduction. In: Bahnson RR, editor. Management of urologic disoreders. Chicago: MosbyYear Book; 1994. p. 28-37.
14. Devroey P, Liu J, Nagy Z, Goossens A, Tournaye H, Camus M, et al. Pregnancies after testicular sperm extraction and intracytoplasmic sperm injection in non-obstructive azoospermia. Hum Reprod 1995;10:1457-60.

15. Matsumiya K, Namiki M, Takahara S, Kondoh N, Takada S, Kiyohara H, et al. Clinical study of azoospermia. Int J Androl 1994;17: 140-2.

16. Van Steirteghem AC, Nagy Z, Joris H, Liu J, Staessen C, Smitz J, et al. High fertilization and implantation rates after intracytoplasmic sperm injection. Hum Reprod 1993;8:1061-6.

17. Silber SJ, Van Steirteghem AC, Liu J, Nagy Z, Tournaye H, Devroey P. High fertilization and pregnancy rate after intracytoplasmic sperm injection with spermatozoa obtained from testicle biopsy. Hum Reprod 1995;10:148-52.

18. Gorelick J, Goldstein M. Loss of fertility in men with varicocele. Fertil Steril 1993;59:613-6.

19. Witt MA, Lipshultz LI. Varicocele: a progressive or static lesion? Urology 1993;42:541-3.

20. MacLeod J. Seminal cytology in the presence of varicocele. Fertil Steril 1965;16:735-57.

21. Harrison RM, Lewis RW, Roberts JA. Pathophysiology of varicocele in nonhuman primates: long-term seminal and testicular changes. Fertil Steril 1986;46:500-10.

22. Chehval MJ, Purcell MH. Deterioration of semen parameters over time in men with untreated varicocele: evidence of progressive testicular damage. Fertil Steril 1992;57:174-7.

23. Nagler HM, Martinis FG. Varicocele. In: Lipshultz LI, Howards SS, editors. Infertility in the male. St. Louis: Mosby Year Book; 1997. p. 336-59. 